\title{
Objetivo Europa 2020. \\ La reducción de la pobreza y la exclusión social en España
}

\section{Olga García Luque}

Universidad de Murcia. Departamento de Economía Aplicada

olga@um.es

\section{Úrsula Faura Martínez \\ Matilde Lafuente Lechuga}

Universidad de Murcia. Departamento de Métodos Cuantitativos para la Economía y la Empresa

faura@um.es; mati@um.es

\section{Resumen}

Este trabajo realiza un balance de situación para España ante el objetivo de reducción del riesgo de pobreza o exclusión social, marcado por la Unión Europea dentro de la Estrategia Europa 2020. La información utilizada procede de la Encuesta de Condiciones de Vida, para los años 2009 y 2013, a través del indicador AROPE y sus tres componentes: pobreza, privación material severa y baja intensidad laboral del hogar. Desde una perspectiva territorial, se examina la evolución sufrida por la población en riesgo, estimando la reducción necesaria en cada comunidad autónoma para lograr el retroceso consignado en el objetivo nacional.

Del comportamiento regional seguido hasta el momento, se infieren importantes dificultades para alcanzar el objetivo asumido en la Estrategia Europa 2020, dado que la mayoría de comunidades han incrementado su población en riesgo muy por encima de la cifra estimada en la que debería haberse reducido para lograrlo. Esto implica que el esfuerzo previsto en materia de lucha contra la exclusión social se ve multiplicado, entre dos y cuatro veces, para la mayoría de las regiones. En un contexto dominado por la austeridad presupuestaria, más allá del conflicto evidente para cumplir con el compromiso adoptado, la situación reclama un cambio decidido en las políticas sociales y económicas, reconquistando el principio europeo de promover la cohesión social y territorial.

Palabras clave: Europa 2020, AROPE, región 


\begin{abstract}
Europe 2020 Target: Reduction of Poverty and Social Exclusion in Spain
This paper assesses the Spanish target of reducing the risk of poverty and social exclusion set out in the Europe 2020 Strategy. Data from the Spanish Survey of Living Conditions (Encuesta de Condiciones de Vida) for the year 2009 and 2013 were used, particularly the AROPE indicator and its three components: poverty, material deprivation and low work intensity. The evolution of the at-risk population is examined from a regional perspective and the reduction of poverty and social exclusion required for each region to meet the national target is estimated. The analysis of regional behavior suggests that there are major difficulties to reach the target established in the Europe 2020 Strategy as the population at risk, which should have decreased, has instead increased well above the estimated figure in most autonomous communities. This implies that the expected efforts to combat social exclusion have multiplied two to four times for most regions. In a context dominated by budget austerity, beyond the obvious challenges involved in fulfilling the commitment made, the situation requires a decisive change in social and economic policies to regain the European principle of promoting social and territorial cohesion.
\end{abstract}

Keywords: Europe 2020; AROPE; region

\title{
Sumario
}

\section{Introducción}

2. Debilidades del objetivo 2020 en materia de pobreza y exclusión social

3. Crisis y crecimiento de la población en riesgo de pobreza o exclusión social en Europa
4. Disparidad territorial y objetivo 2020 en materia de pobreza y exclusión social en España

5. Conclusiones

Referencias bibliográficas

\section{Introducción}

El término exclusión social surge al tiempo que tienen lugar las profundas transformaciones sociales y económicas acaecidas en el último cuarto del siglo Xx en las sociedades más avanzadas. Estos cambios (envejecimiento demográfico, erosión del modelo clásico familiar, crecimiento de la inmigración, precariedad laboral, etc.), a los que se une la reestructuración del estado del bienestar, propiciada por la generalización de políticas de corte neoliberal, han ido engrosando los grupos de población en clara desventaja a los que el término de exclusión social se refiere; tendiendo a sustituir a diversas denominaciones más tradicionales (pobres, necesitados, marginados o de clase baja, entre otras). Este proceso se ha visto reforzado por la actual crisis económica, que ha agravado aún más el riesgo de exclusión social de los colectivos más vulnerables, extendiéndolo a su vez a nuevos grupos de población; al tiempo que ha aumentado el grado de incertidumbre sobre el devenir de las políticas sociales, de nuevo mermadas por recortes presupuestarios en aras de la estabilidad fiscal. 
La actuación de la Unión Europea (UE) ha sido determinante en la consolidación del término exclusión social, lo cual ha contribuido a difundirlo y a conceptualizarlo. Esta institución entiende la exclusión social como:

Un proceso que relega a algunas personas al margen de la sociedad y les impide participar plenamente en ella debido a su pobreza, a la falta de competencias básicas y a oportunidades de aprendizaje permanente, o por motivos de discriminación. Esto las aleja de las oportunidades de empleo, percepción de ingresos y educación, así como de las redes y actividades de las comunidades. Tienen poco acceso a los organismos de poder y decisión y, por ello, se sienten indefensos e incapaces de asumir el control de las decisiones que les afectan en su vida cotidiana. (Comisión Europea, 2003: 9)

En sintonía con la evolución seguida en los últimos años, la UE ha definido un nuevo indicador para medir el objetivo en materia de pobreza y exclusión social, incluido en la Estrategia Europa 2020, el indicador AROPE (at risk of poverty or social exclusión), cuyo carácter multidimensional es quizá su mejor atributo.

Dicha estrategia constituye el nuevo marco comunitario de acción para el periodo 2010-2020, en respuesta a la crisis económica, y se enuncia en cinco objetivos tendentes a lograr un crecimiento económico inteligente, sostenible e integrador. En relación con este último aspecto, dirigido a promover la cohesión social y territorial, se establece el compromiso de reducir la población en riesgo de pobreza y exclusión social en 20 millones de personas (Comisión Europea, 2010a: 10).

La mención a la cohesión territorial no se concreta en ninguna medida en la Estrategia Europa 2020, formulándose con carácter general para su aplicación en el ámbito estatal. Sin embargo, las disparidades territoriales en el seno de la UE son de tal envergadura que el factor espacial no debe ignorarse, sino más bien incorporarse, tanto en el análisis de los objetivos establecidos como en el desarrollo de las políticas dirigidas a su logro, debiéndose implementar actuaciones a escala regional (Lois et al., 2013). Este mismo punto de vista es sostenido por el Comité de las Regiones (2015), que viene reclamando la necesidad de reorientar la Estrategia dotándola de una dimensión territorial. En este sentido, resulta imprescindible un mayor conocimiento de la realidad regional, sobre todo en el ámbito de la exclusión social y, particularmente, en nuestro país, donde «las disparidades regionales contribuyen también al nivel global de desigualdad», según indica la Comisión Europea (2016: 68) en su último informe sobre España, emitido dentro del semestre europeo de coordinación de las políticas económicas.

Todo ello constituye el marco de referencia de este trabajo, cuyo propósito es realizar un balance de la situación de España ante el objetivo europeo de reducción del riesgo de pobreza o exclusión social, adoptando para ello una perspectiva de análisis regional. Las dificultades para alcanzar dicho objetivo se han hecho patentes para el conjunto de la UE como consecuencia de la crisis económica, especialmente para España, por ser uno de los estados europeos 
donde más se ha incrementado la población en riesgo. Además, en nuestro país, las disparidades entre regiones en los niveles de bienestar son muy importantes (OECD, 2014), destacando las divergencias en el riesgo de pobreza o exclusión social (EAPN, 2015; Jurado y Pérez, 2014), lo que dota de mayor interés al enfoque territorial aquí adoptado.

Con la información procedente de la Encuesta de Condiciones de Vida (ECV), se analizan las desigualdades regionales en el indicador AROPE y sus tres componentes: pobreza, privación material severa y baja intensidad laboral del hogar. El periodo de estudio comprende los años 2009 y 2013, referidos a la nueva serie de la ECV tras su cambio metodológico, consistente en la estimación de la renta a partir de fuentes administrativas.

En primer lugar, se revisa el compromiso europeo en materia de pobreza y exclusión social, señalando sus principales limitaciones y describiendo la situación de los países miembros de la UE en relación con su cumplimiento. A continuación, se analiza la evolución experimentada por el riesgo de pobreza y exclusión social en España y sus comunidades autónomas. Posteriormente, se estima la reducción necesaria de la población regional en riesgo para lograr la disminución comprometida en el objetivo nacional de la Estrategia, finalizando con la presentación de las conclusiones principales.

\section{Debilidades del objetivo 2020 en materia de pobreza y exclusión social}

El hecho de que la UE haya establecido por primera vez un objetivo cuantificable en materia de pobreza y exclusión social, formando parte de la Estrategia Europa 2020 (EE2020), constituye un hito importante. Sin embargo, dicho objetivo adolece de ciertas debilidades relacionadas con el escaso grado de compromiso político mostrado en su desarrollo hasta ahora, con el indicador utilizado para medirlo y con la definición del propio objetivo en el ámbito espacial. Este último aspecto permite considerar la relevancia del enfoque territorial en el cumplimiento del objetivo, como se muestra más adelante, al proponer una posible evaluación del mismo referida al caso español.

\subsection{Compromiso político}

La EE2020 constituye la reformulación actualizada al nuevo contexto institucional y de crisis económica de la Estrategia de Lisboa, que gobernó a la actuación político-económica de la Unión Europea entre 2000 y 2010. La ampliación europea hacia los países del Este (iniciada en 2004) y el nuevo marco institucional establecido en el Tratado de Lisboa (firmado a finales de 2007) se unen al estallido de la crisis económica en 2008 y al propio agotamiento de la Estrategia de Lisboa, reclamando compromisos renovados por parte de los estados miembros que impulsen no solo el proceso de integración, sino también que se haga frente a los retos planteados en la maltrecha economía europea, fuertemente impactada por la crisis. 
La EE2020 proporciona, por tanto, el nuevo marco de acción común en la UE para el periodo 2010-2020, heredando de la anterior Estrategia de Lisboa el énfasis por el objetivo de crecimiento y el establecimiento de indicadores medibles del progreso alcanzado (Lois et al., 2013). Además, emulando lo sucedido con su predecesora, hay razones de peso que auguran un nuevo fracaso estratégico una vez recorrida la mitad del horizonte temporal marcada en la misma.

En su enunciado más abreviado y conocido, la EE2020 propone lograr un crecimiento inteligente a lo largo de estos años, promoviendo el conocimiento y la innovación; un crecimiento sostenible, teniendo en cuenta el medio ambiente, y un crecimiento integrador, desde el punto de vista social (Comisión Europea, 2010a: 10). Estas tres prioridades están interrelacionadas y se refuerzan mutuamente mediante la consecución, con meta en el año 2020, de cinco objetivos principales:

1. Conseguir una tasa de empleo del $75 \%$ de la población de 20 a 64 años.

2. Invertir en $\mathrm{I}+\mathrm{D}$ el $3 \%$ del PIB.

3. Reducir las emisiones de gases de efecto invernadero un $20 \%$, incrementando en idéntico porcentaje la eficacia energética y la proporción de energías renovables en el consumo final de energía.

4. Reducir al $10 \%$ el porcentaje de abandono escolar y aumentar al $40 \%$ el porcentaje de población, entre 30 y 34 años, con estudios superiores.

5. Reducir en 20 millones la población europea en riesgo de pobreza o exclusión social.

Sobre el eje prioritario del crecimiento integrador, inciden tanto las medidas dirigidas a la consecución del objetivo de pobreza como las orientadas a los logros en materia de empleo y educación, buscando promover la cohesión social y territorial en el seno de la UE. Por primera vez, se visibiliza la lucha contra la pobreza y la exclusión social en la agenda política de la UE, concretándose su intervención a través de la Plataforma Europea contra la Pobreza y la Exclusión Social, que constituye una de las siete iniciativas emblemáticas o programas de actuación, por medio de los cuales la Unión impulsa la consecución de los cinco objetivos estratégicos. Sin embargo, no hay en la EE2020 un compromiso financiero firme con las políticas activas de inclusión (Arriola, 2012 y 2014), quedándose en propuestas de actuación para ser ejecutadas por los estados miembros, auspiciadas por una mayor coordinación de los fondos estructurales (Comisión Europea, 2010b y 2013a), entre los que cobra protagonismo el Fondo Social Europeo (Unión Europea, 2013). De hecho, el objetivo en materia de pobreza y exclusión es el gran olvidado en los programas nacionales de reformas (PNR), marcados por la austeridad de las cuentas públicas, así como en la revisión de los mismos por parte de las autoridades europeas, donde cobran protagonismo las recomendaciones liberalizadoras y las medidas de estabilidad macroeconómica y presupuestaria (MINHAP, 2015; Comisión Europea, 2015a). 
El problema de fondo, como señala Arriola (2014), es la indefinición de las políticas comunitarias que se debaten, en permanente contradicción, entre las prioridades del ajuste presupuestario y salarial (en aras de una mayor competitividad que impulse el crecimiento económico) y las exigencias de una política efectiva de reducción de la pobreza y la exclusión, que requiere, al menos, de mejores prestaciones sociales y salarios. En esta pugna entre objetivos - los proclives a la eficiencia de los mercados y los orientados hacia la integración social-, los primeros predominan claramente sobre los segundos.

En este sentido, el énfasis en las medidas dirigidas al mercado de trabajo, como principal vía para luchar contra la pobreza y la exclusión social, es evidente en el último PNR de España (MINHAP, 2015). Sin embargo, la capacidad otorgada al crecimiento económico y a la creación de empleo como mecanismos correctores de la pobreza y la exclusión queda desmentida por la trayectoria seguida en nuestro país durante la etapa expansiva previa a la crisis, donde los incrementos de la producción y el empleo se vieron acompañados de aumentos en la desigualdad y del mantenimiento de las tasas de pobreza (Ayala, 2010; Hernández, 2008; Jurado y Pérez, 2010). El cambio de coyuntura económica se ha traducido en un agravamiento de la situación en materia de desigualdad, pobreza y exclusión social, cuestión que ha ser analizada desde un punto de vista estructural, en relación con la evolución que se viene registrando en las últimas décadas en los sistemas económico y de bienestar, en claro avance hacia la precarización de las relaciones laborales, en el caso del primero, y hacia el debilitamiento redistributivo, en el del segundo (Fundación FOESSA, 2014). En consecuencia, la crisis no ha hecho más que evidenciar las tendencias del modelo socioeconómico que se viene consolidando desde principios de la década de 1980, cuyos efectos, agudizados por la recesión económica, han dado como resultado el aumento de las situaciones de vulnerabilidad y exclusión social en España desde 2008, lo cual ha modificado los perfiles de riesgo, al afectar a grupos de población que, con anterioridad, disfrutaban de una situación "normalizada" (Laparra y Pérez, 2012; Lafuente y Faura, 2012; Fundación FOESSA, 2014; Hernández, 2014).

Precisamente, este divorcio entre las condiciones de vida de los colectivos más vulnerables y las políticas económica y social imperantes, observable en el trabajo de intervención desarrollado cotidianamente por las organizaciones del tercer sector, motiva críticas y llamadas continuas de atención sobre el escaso grado de compromiso gubernamental, nacional y europeo, para atajar el problema. En el contexto de la implementación de la EE2020, numerosos informes elaborados por diversas ONG (Cáritas, 2013 y 2014; Cruz Roja, 2013; EAPN, 2013 y 2014), así como estudios realizados desde el ámbito académico (Martínez, 2011; Ayala, 2012; Fresno et al., 2012; Leschke et al., 2012; Frazzer et al., 2014), reclaman la necesidad de reorientar las prioridades europeas hacia la integración social, poniendo en el centro de la EE2020 la lucha contra la pobreza y la exclusión para promover el crecimiento. En oposición a las tesis dominantes, estos análisis advierten sobre los importantes 
costes de oportunidad en términos de ingresos privados y públicos perdidos, además de los relacionados con el deterioro del capital humano, el incremento de la conflictividad social o los efectos intergeneracionales que acarrean unos elevados niveles de desigualdad y pobreza, lo cual incide negativamente sobre la competitividad y el crecimiento (Frazzer et al., 2014).

En medio de esta controversia, la EE2020 ha sido objeto de revisión cuando se encuentra a mitad de camino del horizonte marcado (Comisión Europea, $2014 \mathrm{a}$ y 2014b) y ha obtenido un claro suspenso en materia de empleo, investigación y desarrollo y reducción de la pobreza. Formando parte de este proceso de revisión, recientemente, se han hecho públicos los resultados de la consulta efectuada para recabar opiniones acerca del diseño y los logros alcanzados por la EE2020 hasta el momento (Comisión Europea, 2015b), percibiéndose como una amenaza al cumplimiento de lo pactado, la ampliación de las divergencias entre y dentro de los países miembros durante estos últimos años, así como el carácter no vinculante de las metas propuestas.

\subsection{Indicador seleccionado}

El nuevo indicador propuesto para medir la incidencia del riesgo de pobreza o exclusión social es la tasa AROPE (en adelante, arope), que adopta una concepción multidimensional de la exclusión social y recoge situaciones de vulnerabilidad en las cuales la pobreza es solo uno de los aspectos relevantes. Así, la tasa arope combina tres conceptos: pobreza, privación material severa y baja intensidad de trabajo del hogar.

La primera de estas situaciones, la pobreza, consiste en disponer de unos ingresos inferiores al $60 \%$ de la renta mediana nacional por unidad de consumo equivalente, lo que constituye el umbral de pobreza. En situación de privación material severa, se encuentran aquellas personas cuyos hogares no pueden permitirse cuatro de nueve ítems de consumo básico, referidos a la capacidad del hogar para afrontar determinados gastos (estrés económico) y disponer de ciertos bienes de consumo duradero. En concreto, dichas carencias son: sufrir retrasos en los pagos relacionados con la vivienda principal (hipoteca o alquiler, recibos de gas, electricidad, comunidad, etc.), o en compras a plazos en los últimos 12 meses; no tener capacidad para afrontar gastos imprevistos, o bien no poder permitirse ir de vacaciones al menos una semana al año, ni realizar una comida de carne, pollo o pescado al menos cada dos días; ni mantener la vivienda a una temperatura adecuada; ni disponer de teléfono; de un automóvil; de un televisor en color y de una lavadora. Por último, la situación de baja intensidad de trabajo del hogar se produce cuando el cociente entre el número de meses trabajados por todos los miembros del hogar en edad de trabajar y el número total de meses que, en teoría, podrían haber trabajado es inferior a 0,20. Así, el indicador arope hace referencia al porcentaje de población que se encuentra en riesgo de pobreza y/o de exclusión social, al cumplir al menos con uno de los tres criterios mencionados: ser pobre y/o sufrir de privación material severa y/o vivir en hogares con baja intensidad laboral. 
Aunque el uso de la tasa arope se ha impuesto debido a su utilización como indicador europeo de la EE2020, ha cosechado diversas críticas derivadas de algunas de sus limitaciones metodológicas. La primera de ellas alude a la diferente referencia temporal de los indicadores incluidos en su cálculo: la pobreza y la baja intensidad laboral del hogar están referidos al año anterior al de la entrevista, mientras que la privación material severa corresponde al mismo año. Debido al retardo de un año en la información captada por estos dos indicadores, su capacidad para proporcionar datos actualizados es escasa, como se ha evidenciado tras el primer impacto de la crisis económica actual (Laparra, 2010). En consecuencia, cuando se estableció el objetivo de la EE2020 en 2010, se realizó en base a datos que aún no reflejaban el impacto de la crisis: el año de referencia para definir el objetivo fue 2008, el más reciente entonces con información disponible, pero dicha información en términos de pobreza y del indicador de empleo correspondió al año anterior, 2007. Un problema similar se tendrá en el momento de realizar la evaluación final: no se dispondrá de información correspondiente a 2020 hasta el año 2021 y, con la encuesta de 2020, los datos de pobreza y de baja intensidad laboral irán referidos a 2019. Todo esto plantea diversas dudas acerca de con qué referencia temporal se va a evaluar el objetivo de reducción de la pobreza (Frazzer et al., 2014).

Otra limitación se refiere al ámbito distinto de referencia del umbral para los indicadores de pobreza (renta mediana nacional) y de privación (listado común de nueve ítems de consumo básico definidos para el conjunto de la UE). En la práctica, el indicador de privación resulta ser muy restrictivo para los países de la UE con la renta per cápita más elevada, entre los que se encuentra España, puesto que solo da cuenta de situaciones de privación muy graves (cuatro carencias sobre una lista efectiva de seis indicadores). Además, tres de los bienes duraderos incluidos (televisión, lavadora, teléfono) son ampliamente disfrutados, lo que convierte su carencia en poco relevante. A parte de eso, la elevada proporción de estos consumos duraderos (cuatro de nueve ítems) limita la respuesta del índice de privación a cambios del ciclo económico (Martínez y Navarro, 2014).

Además, los indicadores de pobreza y privación contemplan a toda la población, mientras que el indicador de baja intensidad laboral del hogar se refiere a las personas menores de 60 años, estando excluidos los hogares compuestos solo por niños, estudiantes menores de 25 años y/o personas de 60 años o más. Ward y Ozdemir (2013) señalan diversos inconvenientes de este indicador, los cuales están relacionados con la definición de persona en edad para trabajar o el tratamiento de los estudiantes, entre otros. Por otra parte, el desempleo debería ser considerado, más bien, como un factor que conduce a la pobreza de ingresos o a la privación material y no como un indicador de la pobreza multidimensional en sí mismo (Nolan y Whelan, 2011).

En cualquier caso, pese a estos desajustes entre los distintos indicadores aglutinados en la tasa arope, se hace necesario contrastar su uso debido al protagonismo alcanzado en el contexto europeo. Así, en nuestro país, diversos estudios han dado cuenta de su evolución durante los últimos años, tanto a 
nivel regional como nacional. Entre otros, encontramos a los siguientes: Pérez (2013), Fundación FOESSA (2014), Hernández (2014) y EAPN (2015).

\subsection{Dimensión territorial}

La definición del objetivo europeo en materia de pobreza y exclusión, su traducción a los distintos objetivos nacionales y la ausencia de una dimensión territorial, en la que cobren protagonismo en su desarrollo las regiones y las ciudades europeas, también han recibido distintas críticas.

Quizá la más extendida sea considerarlo escasamente ambicioso. En un primer momento, se enunció referido solo a la población en riesgo de pobreza, que, en 2008, alcanzaba una cifra de 80 millones de personas en el conjunto de la UE: «el número de europeos que viven por debajo de los umbrales nacionales de pobreza debería reducirse en un $25 \%$, rescatando así a más de 20 millones de personas de la pobreza» (Comisión Europea, 2010a: 11). Sin embargo, finalmente, el indicador seleccionado fue la tasa arope, lo que incrementa a la población de referencia y mantiene el valor absoluto de la reducción en 20 millones de personas. De esta manera, el objetivo estratégico en materia de lucha contra la pobreza resultó ser mucho menos ambicioso en la redacción final de la EE2020 (Nolan y Whelan, 2011).

En el contexto actual de crisis y agravamiento de la pobreza, en el que las últimas cifras disponibles para 2013 estiman a la población arope en 123 millones de personas en la UE-28 (Eurostat, 2015a), haber definido su reducción en términos absolutos en lugar de hacerlo en porcentaje, como se ha realizado con el resto de objetivos de la EE2020, refleja un menor compromiso en esta materia. De hecho, cuando se comienzan a divulgar los primeros resultados de la EE2020, el objetivo en materia de reducción de la pobreza es el único cuya trayectoria de consecución no se proporciona, argumentándose que «no se mide de la misma manera que los demás objetivos, debido a la falta de estadísticas comparables» (Comisión Europea 2013a: 5). Dicha argumentación no es fácil de admitir, puesto que la información sobre pobreza y exclusión social procede de una estadística armonizada para todos los países de la UE, la European Union Statistics on Income and Living Conditions (EU-SILC).

La cuestión es otra. La dificultad para comparar las trayectorias seguidas por los distintos países en materia de pobreza tiene que ver con la traducción de la EE2020 a objetivos nacionales para que cada país pueda comprobar su propio progreso. Dicha traducción al ámbito nacional ha supuesto una merma de los objetivos de la EE2020, al tiempo que pone en peligro su cumplimiento desde el punto de vista global, puesto que la suma por países no garantiza la consecución de cada objetivo común, lo que abiertamente ha admitido la Comisión Europea (2014a). Precisamente, el objetivo de reducción del riesgo de pobreza o exclusión social es el más afectado por esta cuestión: varios países establecen su objetivo a partir de un indicador distinto a la tasa arope (Alemania, Dinamarca, Estonia, Holanda, Irlanda, Letonia y Suecia). En el resto, donde el objetivo nacional en materia de pobreza es 
compatible y comparable con el comunitario, el compromiso de reducción de la población en riesgo de pobreza y exclusión social ha descendido de forma considerable ${ }^{1}$. En el caso español, el objetivo de disminución de la población en riesgo de pobreza o exclusión social se concreta en un intervalo de 1,4 a 1,5 millones de personas.

Este problema podría haberse salvado con una mayor voluntad política para preservar el objetivo común, en el momento de definir el propio, por parte de todos los estados miembros. Además, si se hubiese establecido la reducción del riesgo de pobreza o exclusión social en términos relativos y no absolutos, como se ha hecho, se habría tenido en cuenta el punto de partida de cada territorio, favoreciendo la comparabilidad de los resultados y su evaluación en diferentes niveles espaciales. De este modo, las dificultades para analizar el objetivo en materia de pobreza y exclusión social a escala regional o local, derivadas principalmente de la insuficiente información estadística, se ven reforzadas al haberse definido como una reducción del número de personas en riesgo. Así, en los pocos estudios que comparan el grado de cumplimiento de la EE2020 para las regiones europeas, se suele transformar la disminución absoluta de las personas en riesgo de pobreza o exclusión social en un porcentaje de la población total, mediante diversos métodos (Lois et al., 2013; Athanasoglou y Dijkstra, 2014; Dijkstra y Athanasoglou, 2015).

Estas dificultades han llevado al Comité de las Regiones a insistir en la necesidad de diferenciar los objetivos de la EE2020 a escala regional, y no solo nacional. Los escasos logros alcanzados se interpretan en clave de la falta de una dimensión territorial en los compromisos pactados, proponiendo sustituir el esquema actual descendente por otro de gobernanza multinivel, en el que las administraciones regionales y locales desempeñen un papel activo (Comité de las Regiones, 2014). Sus argumentos descansan en la división territorial de poderes en los estados miembros, con amplias competencias de las administraciones locales y regionales en muchos de ellos y en la mayoría de los ámbitos políticos de la EE2020, junto con la actividad desarrollada por los gobiernos regionales en estos años de crisis en favor de los objetivos estratégicos, a pesar de sus complicadas condiciones presupuestarias.

\section{Crisis y crecimiento de la población en riesgo de pobreza o exclusión social en Europa}

Como se ha dicho, la situación en materia de pobreza y exclusión social en el conjunto de la UE se ha visto considerablemente agravada como consecuencia de la crisis económica, lo cual ha augurado el fracaso del objetivo establecido para 2020. El cuadro 1 recoge la evolución registrada entre 2008 y 2013 por el número de personas en riesgo de pobreza o exclusión social en los países de la UE-27, así como sus respectivos porcentajes en términos de la población

1. Los objetivos nacionales pueden consultarse en http://ec.europa.eu/europe2020/europe2020-in-a-nutshell/targets/index_en.htm 
Cuadro 1. Población en riesgo de pobreza o exclusión social en la Unión Europea (2008-2013)

\begin{tabular}{|c|c|c|c|c|c|c|c|c|}
\hline \multirow[b]{3}{*}{ Países } & \multicolumn{3}{|c|}{ Porcentaje sobre el total } & \multicolumn{5}{|c|}{ Miles de personas } \\
\hline & \multirow[b]{2}{*}{2008} & \multirow[b]{2}{*}{2009} & \multirow[b]{2}{*}{2013} & \multirow[b]{2}{*}{2008} & \multirow[b]{2}{*}{2009} & \multirow[b]{2}{*}{2013} & \multicolumn{2}{|c|}{ Variación } \\
\hline & & & & & & & $2008-2013$ & 2009-2013 \\
\hline Alemania & 20,1 & 20,0 & 20,3 & 16.345 & 16.217 & 16.212 & -133 & $-5,0$ \\
\hline Austria & 20,6 & 19,1 & 18,8 & 1.699 & 1.577 & 1.572 & -127 & $-5,0$ \\
\hline Bélgica & 20,8 & 20,2 & 20,8 & 2.194 & 2.145 & 2.286 & 92 & 141,0 \\
\hline Bulgaria & 44,8 & 46,2 & 48,0 & 3.421 & 3.511 & 3.493 & 72 & $-18,0$ \\
\hline Chipre & 23,3 & 23,5 & 27,8 & 181 & 188 & 240 & 59 & 52,0 \\
\hline Dinamarca & 16,3 & 17,6 & 18,9 & 887 & 962 & 1.059 & 172 & 97,0 \\
\hline Eslovaquia & 20,6 & 19,6 & 19,8 & 1.111 & 1.061 & 1.070 & -41 & 9,0 \\
\hline Eslovenia & 18,5 & 17,1 & 20,4 & 361 & 339 & 410 & 49 & 71,0 \\
\hline España & 24,5 & 24,7 & 27,3 & 11.124 & 11.336 & 12.630 & 1.506 & $1.294,0$ \\
\hline Estonia & 21,8 & 23,4 & 23,5 & 291 & 312 & 313 & 22 & 1,0 \\
\hline Finlandia & 17,4 & 16,9 & 16,0 & 910 & 886 & 854 & -56 & $-32,0$ \\
\hline Francia & 18,5 & 18,5 & 18,1 & 11.150 & 11.200 & 11.229 & 79 & 29,0 \\
\hline Grecia & 28,1 & 27,6 & 35,7 & 3.046 & 3.007 & 3.904 & 858 & 897,0 \\
\hline Holanda & 14,9 & 15,1 & 15,9 & 2.432 & 2.483 & 2.648 & 216 & 165,0 \\
\hline Hungría & 28,2 & 29,6 & 33,5 & 2.794 & 2.924 & 3.285 & 491 & 361,0 \\
\hline Irlanda & 23,7 & 25,7 & 29,5 & 1.050 & 1.150 & 1.358 & 308 & 208,0 \\
\hline Italia & 25,3 & 24,7 & 28,4 & 15.099 & 14.835 & 17.326 & 2.227 & $2.491,0$ \\
\hline Letonia & 34,2 & 37,9 & 35,1 & 740 & 808 & 702 & -38 & $-106,0$ \\
\hline Lituania & 28,3 & 29,6 & 30,8 & 910 & 943 & 917 & 7 & $-26,0$ \\
\hline Luxemburgo & 15,5 & 17,8 & 19,0 & 72 & 85 & 96 & 24 & 11,0 \\
\hline Malta & 20,1 & 20,3 & 24,0 & 81 & 82 & 99 & 18 & 17,0 \\
\hline Polonia & 30,5 & 27,8 & 25,8 & 11.491 & 10.454 & 9.748 & -1.743 & $-706,0$ \\
\hline Portugal & 26,0 & 24,9 & 27,5 & 2.757 & 2.648 & 2.879 & 122 & 231,0 \\
\hline Reino Unido & 23,2 & 22,0 & 24,8 & 14.069 & 13.389 & 15.586 & 1.517 & $2.197,0$ \\
\hline República Checa & 15,3 & 14,0 & 14,6 & 1.566 & 1.448 & 1.508 & -58 & 60,0 \\
\hline Rumanía & 44,2 & 43,1 & 40,4 & 9.418 & 9.112 & 8.601 & -817 & $-511,0$ \\
\hline Suecia & 14,9 & 15,9 & 16,4 & 1.367 & 1.459 & 1.602 & 235 & 143,0 \\
\hline Totales UE-271 & 23,8 & 23,3 & 24,5 & 116.566 & 114.560 & 121.626 & 5.060 & $7.066,0$ \\
\hline
\end{tabular}

1. Se considera el agregado UE-27 al no haber datos referidos a UE-28 para los años 2008 y 2009. Para España y el Reino Unido, los datos de 2013 no son estrictamente comparables con los anteriores, al haber una ruptura de la serie en 2012.

Fuente: Eurostat (2015a), Statistics on income, social inclusion and living conditions.

total, es decir, su tasa arope. Además del año 2008, referencia de la EE2020, se muestran los datos correspondientes a 2009 y 2013, puesto que este último intervalo es el que se toma como referencia en el resto de este trabajo, al utilizar la nueva serie de la ECV (base 2013) para el análisis español ${ }^{2}$.

2. El dato de España de 2009 recogido en el cuadro 1 no coincide con los mostrados en los siguientes cuadros, pues las cifras publicadas por Eurostat hasta 2012 corresponden a la metodología anterior de la ECV (base 2004). 
Las tasas arope más altas en 2013 corresponden a Bulgaria (48\%) y Rumanía $(40,4 \%)$. Les siguen Grecia, Letonia y Hungría, con porcentajes superiores a un tercio de la población. España ocupa la undécima peor posición en función de su tasa arope (27,3\%). Los cinco países con menores porcentajes de población en riesgo de pobreza o exclusión social en 2013 son República Checa (14,6\%), Holanda (15,9\%), Finlandia (16,0\%), Suecia $(16,4 \%)$ y Francia $(18,1 \%)$.

El incremento de la población en riesgo de pobreza o exclusión social en nuestro país durante estos años ha sido de los más elevados de la UE (más de un millón de personas), junto a los de Italia y Reino Unido. Por el contrario, Polonia encabeza el listado de países que siguen la trayectoria adecuada, puesto que muestra una importante reducción en sus tasas. El año tomado como referencia para medir la evolución en el número de personas afectadas durante el periodo de crisis es relevante (cuadro 1). La variación experimentada por cada país difiere bastante según se calcule para el periodo 2008-2013 o bien para los años 2009-2013, en función de cuando haya tenido lugar el primer impacto de la crisis y la trayectoria seguida durante la misma. Así, por ejemplo, el incremento de la población arope en nuestro país es superior entre 2008 y 2013 en relación con el experimentado entre 2009 y 2013, sucediendo lo contrario en el Reino Unido. Esto se explica porque, en España, el mínimo de la población en riesgo de pobreza o exclusión social se alcanza en 2007 y aumenta ya en 2008; mientras que, en el conjunto de la UE y para buena parte de los países europeos, este mínimo se alcanza en 2009. De este modo, en la evaluación final del objetivo, el año de referencia seleccionado cobra una importancia especial, pudiendo verse afectado el resultado si las autoridades europeas consideran modificar el usado hasta ahora (2008), como se sugiere en Frazzer et al. (2014).

Tanto la UE como España están muy lejos de alcanzar lo comprometido en materia de pobreza y exclusión social (cuadro 1), puesto que el deterioro de la situación económica ha empujado a caminar en la dirección opuesta, lo cual ha incrementado las divergencias entre los países europeos (la distancia entre el valor máximo y el mínimo de la tasa arope se eleva entre 2008 y 2013).

La evaluación de lo realizado por parte del gobierno español para la consecución de dicho objetivo no es positiva: «Hasta la fecha, se han tomado solo medidas limitadas para abordar la pobreza y los problemas de integración social» (Comisión Europea, 2013b: 14), cuestión que se vuelve a repetir más recientemente: «España ha realizado avances limitados en la mejora de la eficacia de su sistema de protección social» (Comisión Europea, 2015a: 5). La UE, por otro lado, reconoce que se necesita un esfuerzo mayor por parte de todos para reconducir el objetivo en materia de pobreza a la meta pactada para 2020 (Eurostat, 2015b), siendo fundamental establecer medidas de política social dirigidas a los colectivos más vulnerables (niños pobres, jóvenes desempleados o parados de larga duración, entre otros). En este sentido, preocupa que los países reduzcan el número de pobres o en riesgo de exclusión de forma global, sin que mejoren las condiciones de vida de los grupos de 
población más desfavorecidos (inmigrantes, personas sin hogar, colectivo gitano) (Frazzer et al., 2014).

\section{Disparidad territorial y objetivo 2020 en materia de pobreza y exclusión social en España}

En nuestro país, el proceso de descentralización del gasto social hacia las comunidades autónomas y las divergencias socioeconómicas entre ellas otorgan una relevancia especial a los factores de tipo espacial. Así, la relevancia de las denominadas "causas territoriales de la pobreza», derivadas de una distribución regional dispar de los factores educativos, laborales o demográficos (Jurado y Pérez, 2010: 62-63), se ve reafirmada por el carácter descentralizado de la política social.

En este epígrafe, se examina, por un lado, la evolución de la población en riesgo de pobreza o exclusión social para las comunidades autónomas españolas entre 2009 y 2013. Por otro lado, se estima la reducción necesaria de la población arope de cada región para lograr el retroceso consignado en el objetivo nacional. Se han considerado las diecisiete autonomías españolas, a las que se añaden de forma agregada las ciudades autónomas de Ceuta y Melilla.

La ECV proporciona información de la tasa arope referida al ámbito regional, si bien el tamaño de la muestra autonómica a menudo resulta insuficiente para caracterizar de forma adecuada a la población regional en riesgo de pobreza o exclusión social. En algunas regiones, esta circunstancia afecta incluso al cálculo de la incidencia de alguno de los componentes de la tasa arope, principalmente al indicador de privación material severa. En 2009, la mitad de las comunidades españolas (nueve) cuentan con un reducido número de observaciones (inferior a 50) para determinar a la población en situación de privación material severa ${ }^{3}$, lo cual afecta a la evolución temporal de dicho indicador e influye también en la del agregado arope.

\subsection{Trayectoria durante la crisis: aumento de la población en riesgo de pobreza o exclusión social}

Para conocer cuál ha sido la contribución de las distintas comunidades autónomas al crecimiento experimentado por la población en riesgo de pobreza o exclusión social durante estos años de crisis, se debe partir de la importancia relativa que cada región tiene en términos de la población arope en 2009, por un lado, y de la variación registrada por dicha población durante el periodo considerado, por otro. El producto de ambos factores — peso en el año inicial y tasa de variación regional- determina la contribución regional al crecimiento de la población arope entre 2009 y 2013. Así, la tasa de variación del total de la población española en riesgo de pobreza o exclusión es la suma de las con-

3. En 2013, el número de autonomías con este problema se reduce a cinco, pero sigue siendo una cantidad considerable. 
Cuadro 2. Contribuciones regionales al crecimiento de la población en riesgo de pobreza o exclusión social en España (2009-2013)

\begin{tabular}{|c|c|c|c|c|c|c|}
\hline \multirow{2}{*}{$\begin{array}{l}\text { Comunidad } \\
\text { autónoma }\end{array}$} & \multicolumn{2}{|c|}{ Población arope } & \multirow{2}{*}{$\begin{array}{c}\text { Ponderación }{ }^{1} \\
2009 \\
\end{array}$} & \multirow{2}{*}{$\begin{array}{c}\text { TVP (\%) } \\
2009-2013\end{array}$} & \multicolumn{2}{|c|}{ Contribución ${ }^{2}$} \\
\hline & 2009 & 2013 & & & (p. p.) & $(\%)$ \\
\hline Andalucía & 2.725 .663 & 3.194 .745 & 0,240 & 17,2 & 4,1 & 36,2 \\
\hline Aragón & 167.451 & 259.897 & 0,015 & 55,2 & 0,8 & 7,3 \\
\hline Asturias & 201.746 & 229.792 & 0,018 & 13,9 & 0,3 & 2,2 \\
\hline Baleares & 258.413 & 305.242 & 0,023 & 18,1 & 0,4 & 3,7 \\
\hline Canarias & 767.117 & 746.075 & 0,068 & $-2,7$ & $-0,2$ & $-1,6$ \\
\hline Cantabria & 103.816 & 147.659 & 0,009 & 42,2 & 0,4 & 3,3 \\
\hline Castilla-La Mancha & 584.493 & 767.102 & 0,052 & 31,2 & 1,6 & 14,2 \\
\hline Castilla y León & 556.109 & 512.043 & 0,049 & $-7,9$ & $-0,4$ & $-3,4$ \\
\hline Cataluña & 1.397 .261 & 1.488 .188 & 0,123 & 6,5 & 0,8 & 7,0 \\
\hline Ceuta y Melilla & 52.979 & 65.644 & 0,005 & 23,9 & 0,1 & 1,0 \\
\hline C. Valenciana & 1.304 .378 & 1.561 .437 & 0,115 & 19,7 & 2,3 & 19,9 \\
\hline Extremadura & 382.697 & 389.262 & 0,034 & 1,7 & 0,1 & 0,5 \\
\hline Galicia & 679.428 & 663.655 & 0,060 & $-2,3$ & $-0,1$ & $-1,2$ \\
\hline La Rioja & 61.235 & 69.801 & 0,005 & 14,0 & 0,1 & 0,6 \\
\hline Madrid & 1.232 .457 & 1.277 .264 & 0,109 & 3,6 & 0,4 & 3,4 \\
\hline Navarra & 64.472 & 91.621 & 0,006 & 42,1 & 0,3 & 2,2 \\
\hline País Vasco & 315.587 & 364.470 & 0,028 & 15,5 & 0,4 & 3,8 \\
\hline R. de Murcia & 480.420 & 496.492 & 0,042 & 3,3 & 0,1 & 1,2 \\
\hline Totales España & 11.335 .722 & 12.630 .389 & 1,000 & 11,4 & 11,4 & 100,0 \\
\hline
\end{tabular}

1. Peso regional en la población arope nacional en 2009.

2. Contribución regional en puntos porcentuales (p. p.) al crecimiento nacional de la población arope, resultado de multiplicar el peso regional por su tasa de variación. Se puede expresar también en porcentaje. En todos los cálculos, se han redondeado decimales.

Fuente: elaboración propia a partir de INE (2015), Encuesta de Condiciones de Vida.

tribuciones regionales a dicha variación expresadas en puntos porcentuales, y también se pueden presentar en porcentaje, como muestra el cuadro 2.

Es de esperar que Andalucía sea la región que más contribuya al crecimiento en España de la población en riesgo de pobreza o exclusión social, como efectivamente sucede entre 2009 y 2013 (4,1 puntos porcentuales), pero no solo por su mayor peso relativo en la población arope nacional (un 24\%), sino también debido al elevado crecimiento regional (un 17,2\%), superior al registrado por el total (un 11,4\%). Esto significa que más de un tercio (un $36,2 \%$ ) de la tasa de variación de la población arope española durante estos años viene explicada por el avance en la región andaluza de esta población. A mayor distancia, la Comunidad Valenciana y Castilla-La Mancha contribuyen también de forma importante al crecimiento de la población en riesgo de pobreza o exclusión social.

Precisamente, las dos autonomías castellanas, de similar peso poblacional, registran sin embargo contribuciones muy diferentes (cuadro 2), debido a la dispar evolución que experimentan ambas en el periodo, ya que en Castilla y 
Cuadro 3. Tasas regionales de riesgo de pobreza o exclusión social (2009-2013)

\begin{tabular}{|c|c|c|c|c|c|c|c|}
\hline \multirow{2}{*}{$\begin{array}{l}\text { Comunidad } \\
\text { autónoma }\end{array}$} & \multicolumn{2}{|c|}{ \% población } & \multicolumn{2}{|c|}{ España $=100$} & \multicolumn{3}{|c|}{ Ranking CCAA (ascendente) } \\
\hline & 2009 & 2013 & 2009 & 2013 & 2009 & 2013 & 2013-2009 \\
\hline Andalucía & 32,7 & 39,1 & 133,3 & 141,8 & 15 & 17 & 2 \\
\hline Aragón & 12,7 & 19,6 & 52,0 & 71,3 & 2 & 3 & 1 \\
\hline Asturias & 19,2 & 21,4 & 78,2 & 77,9 & 6 & 7 & 1 \\
\hline Baleares & 23,6 & 28,7 & 96,2 & 104,2 & 10 & 11 & 1 \\
\hline Canarias & 36,5 & 37,2 & 149,1 & 135,0 & 18 & 15 & -3 \\
\hline Cantabria & 17,8 & 24,6 & 72,6 & 89,5 & 4 & 10 & 6 \\
\hline Castilla-La Mancha & 27,9 & 37,5 & 114,0 & 136,1 & 13 & 16 & 3 \\
\hline Castilla y León & 22,6 & 20,4 & 92,4 & 74,0 & 9 & 6 & -3 \\
\hline Cataluña & 18,9 & 20,3 & 77,1 & 73,6 & 5 & 4 & -1 \\
\hline Ceuta y Melilla & 32,1 & 44,3 & 131,0 & 160,8 & 14 & 18 & 4 \\
\hline C. Valenciana & 26,5 & 31,7 & 107,9 & 115,0 & 12 & 12 & 0 \\
\hline Extremadura & 35,4 & 36,0 & 144,6 & 130,7 & 17 & 14 & -3 \\
\hline Galicia & 24,9 & 24,0 & 101,6 & 87,2 & 11 & 9 & -2 \\
\hline La Rioja & 19,4 & 22,3 & 79,3 & 80,9 & 8 & 8 & 0 \\
\hline Madrid & 19,4 & 20,3 & 79,2 & 73,7 & 7 & 5 & -2 \\
\hline Navarra & 10,2 & 14,8 & 41,5 & 53,9 & 1 & 1 & 0 \\
\hline País Vasco & 14,6 & 17,1 & 59,5 & 62,1 & 3 & 2 & -1 \\
\hline R. de Murcia & 33,0 & 34,4 & 134,7 & 125,0 & 16 & 13 & -3 \\
\hline Totales España & 24,5 & 27,5 & 100,0 & 100,0 & - & - & - \\
\hline
\end{tabular}

Fuente: elaboración propia a partir de INE (2015), Encuesta de Condiciones de Vida.

León la población arope se reduce un 7,9\%, mientras que Castilla-La Mancha es de las comunidades donde más aumenta (un 31,2\%). El crecimiento más elevado de la población arope tiene lugar en Aragón (un 55,2\% entre 2009 y 2013), lo que conlleva que esta región aporte a la variación nacional lo mismo que Cataluña, cuyo peso poblacional es bastante superior.

De este modo, conviene tener en cuenta que autonomías con pesos poblacionales similares (como Cataluña, Comunidad Valenciana o Madrid, o también Castilla-La Mancha y Castilla y León) han contribuido de forma muy diferente al avance de la población en riesgo de pobreza o exclusión social en España durante la crisis, debido a sus propias dinámicas regionales; mientras que, por el contario, una evolución regional parecida (en el caso de Baleares y Andalucía, por ejemplo) ha tenido una repercusión distinta sobre el aumento global del riesgo de pobreza o exclusión, en función del tamaño relativo de la población arope regional. Tales consideraciones son relevantes para orientar las medidas dirigidas a lograr el objetivo español en materia de pobreza y exclusión social comprometido en la EE2020, que debe tener en cuenta su dimensión territorial, debido a la importante disparidad regional existente.

La desigualdad territorial en España queda patente al examinar la incidencia dispar que el riesgo de pobreza o exclusión social alcanza entre las comunidades autónomas (cuadro 3). En ambos años, las tasas arope de cinco autonomías 
Figura 1. Variaciones regionales de la tasa arope expresadas en porcentajes (2009-2013)

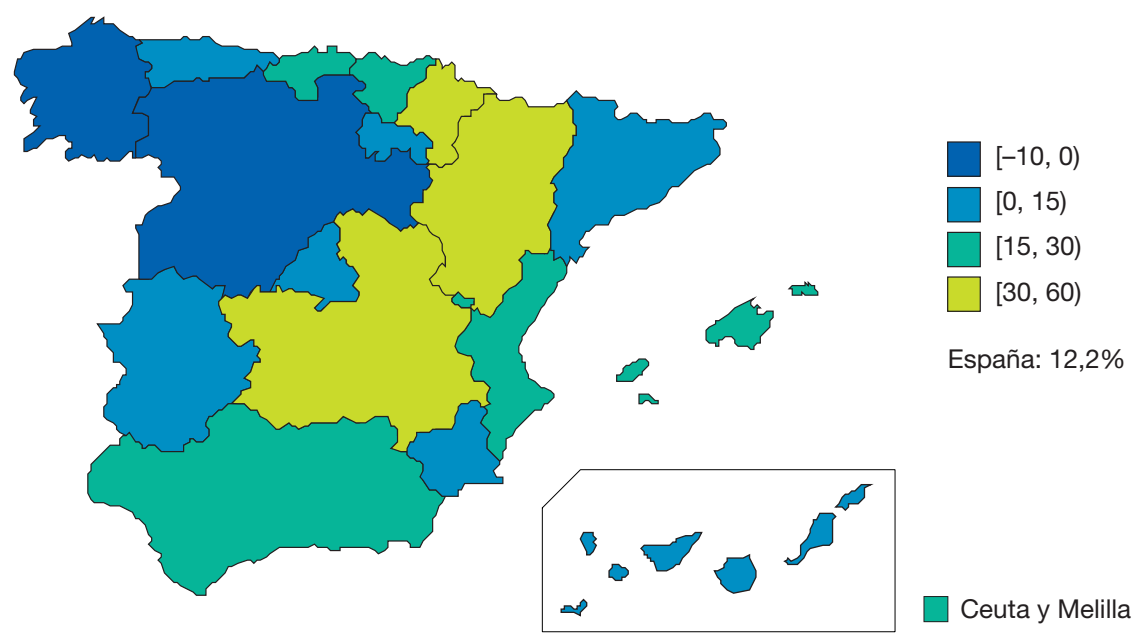

Fuente: elaboración propia a partir de INE (2015), Encuesta de Condiciones de Vida.

del sur superan ampliamente al promedio español: Ceuta y Melilla, Andalucía, Castilla-La Mancha, Canarias y Extremadura. En el extremo opuesto, tres comunidades del norte registran valores de la tasa arope bastante inferiores a la media: Navarra, País Vasco y Aragón. Estas comunidades mantienen una distancia importante con respecto al conjunto nacional, del orden de un 30\% o más, por encima o por debajo de dicho promedio. La divergencia es también relevante para Asturias, Castilla y León, Cataluña, La Rioja y Madrid, con tasas inferiores entre un 20 y un 25\% a la media nacional, así como para la Región de Murcia, situada en la posición contraria. El resto de regiones (Baleares, Cantabria, Comunidad Valenciana y Galicia) se ubica en entornos más cercanos al total nacional, sobre todo en 2013.

Las autonomías que encabezan y cierran la lista se han mantenido sin grandes alteraciones durante el periodo 2009-2013, siendo Cantabria la que más ha empeorado su posición relativa, al pasar del cuarto al décimo lugar, según el ranking de menor a mayor tasa arope. Sin embargo, ello no debe ocultar el impacto territorial desigual que la crisis ha promovido sobre el comportamiento de la tasa arope, que ha aumentado de forma generalizada, aunque no con la misma intensidad, en todas las comunidades autónomas salvo en Galicia y Castilla y León, donde se ha reducido (figura 1).

Como resultado, vemos que la mayoría de regiones incrementa su riesgo relativo de pobreza o exclusión social, ya sea porque reducen su distancia con respecto a la media nacional partiendo de una situación relativamente favorable (como Aragón) o bien porque acrecientan dicha distancia desde una situación claramente desfavorable (Andalucía, por ejemplo). 
Cuadro 4. Componentes regionales de la tasa arope en el período 2009-2013 (\% población total)

\begin{tabular}{|c|c|c|c|c|c|c|}
\hline \multirow{2}{*}{$\begin{array}{l}\text { Comunidad } \\
\text { autónoma }\end{array}$} & \multicolumn{2}{|c|}{ Pobreza } & \multicolumn{2}{|c|}{ Privación material } & \multicolumn{2}{|c|}{ Baja intensidad laboral } \\
\hline & 2009 & 2013 & 2009 & 2013 & 2009 & 2013 \\
\hline Andalucía & 28,8 & 29,1 & 5,7 & 7,0 & 11,4 & 25,3 \\
\hline Aragón & 11,3 & 16,1 & 0,2 & 3,3 & 2,6 & 10,2 \\
\hline Asturias & 13,0 & 14,1 & 1,4 & 4,2 & 8,7 & 16,5 \\
\hline Baleares & 18,1 & 19,8 & 6,3 & 10,4 & 5,0 & 7,1 \\
\hline Canarias & 30,4 & 28,4 & 12,7 & 8,5 & 12,0 & 22,8 \\
\hline Cantabria & 13,9 & 17,8 & 1,2 & 3,9 & 6,8 & 11,1 \\
\hline Castilla-La Mancha & 24,8 & 31,3 & 3,8 & 5,8 & 6,0 & 19,9 \\
\hline Castilla y León & 18,8 & 17,5 & 2,1 & 1,8 & 8,3 & 12,8 \\
\hline Cataluña & 15,2 & 13,9 & 3,1 & 6,1 & 5,6 & 10,8 \\
\hline Ceuta y Melilla & 33,4 & 32,0 & 5,7 & 15,1 & 19,8 & 22,8 \\
\hline C. Valenciana & 20,9 & 23,6 & 5,5 & 7,1 & 10,6 & 16,9 \\
\hline Extremadura & 30,9 & 30,9 & 1,3 & 3,7 & 7,9 & 17,8 \\
\hline Galicia & 20,6 & 17,2 & 3,4 & 4,6 & 5,1 & 14,1 \\
\hline La Rioja & 17,2 & 19,3 & 1,7 & 6,6 & 5,1 & 10,4 \\
\hline Madrid & 15,9 & 13,4 & 4,7 & 7,3 & 3,8 & 9,3 \\
\hline Navarra & 7,8 & 9,9 & 2,1 & 0,6 & 5,5 & 8,0 \\
\hline País Vasco & 10,0 & 10,5 & 3,4 & 4,8 & 7,6 & 13,0 \\
\hline R. de Murcia & 29,1 & 26,8 & 8,2 & 9,0 & 11,4 & 17,3 \\
\hline Totales España & 20,4 & 20,4 & 4,5 & 6,2 & 7,6 & 15,7 \\
\hline
\end{tabular}

Fuente: elaboración propia a partir de INE (2015), Encuesta de Condiciones de Vida.

Las posiciones regionales resultantes de la tasa arope reflejan en gran medida las que se derivan de las tasas de pobreza, que es el componente que determina un mayor porcentaje de la población en riesgo de pobreza o exclusión social (cuadro 4).

Entre 2009 y 2013, la tasa de pobreza nacional se ha mantenido estable, incluso se ha reducido en algunas comunidades. Esto quizá se deba a la influencia del cambio metodológico de la ECV (Vega y Méndez, 2014), dado que diversos trabajos, basados en los datos de la serie anterior, hasta 2012, constataban un incremento moderado de la pobreza para el conjunto nacional durante la crisis, aunque también se observaban descensos para algunas regiones (Jurado y Pérez, 2014; EAPN, 2015).

La disminución del umbral de pobreza, debido al retroceso de la renta mediana española durante la crisis ${ }^{4}$, ha propiciado la salida de la pobreza de

4. Para evitar este efecto derivado del cambio en el ciclo económico, se suele calcular la tasa de pobreza anclando el umbral en un año determinado y actualizándolo en años sucesivos en función de los precios. Calculada de esta manera, la tasa de pobreza nacional registra un crecimiento más intenso en los años de crisis que el mostrado en términos nominales, y todas las regiones incrementan sus tasas de pobreza entre 2008 y 2012 (Jurado y Pérez, 2014: 21). 
determinados grupos de población situados por debajo de dicho umbral, pero muy próximos a él. Un ejemplo claro lo constituye la población de mayores de 65 años, que experimenta una mejoría muy importante de su tasa de pobreza durante la crisis, tanto en el ámbito nacional (Fundación FOESSA, 2014; EAPN, 2015) como regional (Hernández, 2014). Esto no sucede así por la mejora de su situación en términos absolutos, sino en términos relativos, a causa de la mayor estabilidad de sus ingresos frente a la caída de los mismos para el resto de grupos de población.

Sin duda, la menor proporción de pobres entre los mayores de 65 años influye en los descensos experimentados en las tasas regionales de pobreza de las comunidades más envejecidas, como Galicia y Castilla y León. En cambio, para el resto de regiones, cuyas tasas de pobreza disminuyen entre 2009 y 2013 (Canarias, Cataluña, Ceuta y Melilla, Madrid y Región de Murcia), el envejecimiento no parece una causa plausible. En cualquier caso, la mayoría de las regiones experimentan crecimientos de su tasa de pobreza, siendo especialmente elevados en Aragón, Cantabria y Castilla-La Mancha.

En oposición al comportamiento, en general, más moderado de la pobreza, los otros dos componentes de la tasa arope se muestran mucho más expansivos, sobre todo el indicador de baja intensidad laboral, como consecuencia de la fuerte destrucción de empleo en nuestro país durante estos años, que se duplicó para el conjunto nacional y gran parte de las regiones. Por su parte, la privación material severa registra un incremento durante el periodo del $37,8 \%$ para el conjunto nacional, aumentando de forma dispar en casi todas las comunidades a excepción de Canarias, Castilla y León y Navarra. La evolución del indicador de privación debe tomarse con cautela en aquellas comunidades con problemas de representatividad de la muestra en alguno de los dos años considerados, puesto que evidencia variaciones excepcionalmente elevadas y dispersas con respecto al total nacional. No obstante, aunque las limitaciones regionales de la muestra influyan no solo en el análisis de cada territorio, sino también de forma indirecta en el dato global, no cabe duda de que estos resultados apuntan a que la crisis ha incrementado de forma notable las carencias materiales en España.

En consecuencia, con la crisis económica, la mayoría de las comunidades que partían de niveles inferiores a la media nacional en alguno de los tres componentes de la tasa arope ven erosionado este menor riesgo relativo de pobreza o exclusión social. De entre ellas, destacan Aragón y País Vasco, por sufrir dicho proceso en todos los indicadores. Por otro lado, las autonomías que se ubican en las peores posiciones relativas en uno o más de los indicadores mantienen dicha ubicación, aunque reduzcan ligeramente su desventaja, como Canarias; no tanto por una mejora de su situación, sino más bien porque el deterioro nacional de las condiciones materiales de vida, de empleo y de pobreza ha sido superior al regional, sobre todo cuando este parte de niveles mucho más elevados. No obstante, dentro de este grupo de regiones del sur con elevado riesgo relativo de pobreza o exclusión social, Andalucía y Castilla-La Mancha agravan aún más su situación, al empeorar sus indicadores en mayor medida que los promedios nacionales respectivos. 


\subsection{Trayectoria hacia 2020: ¿reducción del riesgo de pobreza o exclusión social?}

Dado el incremento del riesgo de pobreza y exclusión social en España entre 2009 y 2013, cabe plantearse qué posibilidades hay de reconducir la evolución de la población arope hacia el objetivo marcado en la EE2020. Para tratar de dar una respuesta a esta cuestión, proporcionando una hipotética medición del esfuerzo requerido, se ha realizado un sencillo ejercicio de cálculo que permite respetar la reducción del número de personas en riesgo de pobreza o exclusión social establecida en el objetivo nacional. Dado que la comparativa se limita a las regiones españolas, es posible evitar tener que expresar el objetivo nacional en términos relativos, como sucede cuando el análisis se amplía a las distintas regiones europeas, con lo que se consigue una mayor simplicidad en los cálculos y en la interpretación de los resultados obtenidos (cuadro 5).

El planteamiento de partida ha sido considerar la consecución del objetivo nacional en su versión más modesta, o menos ambiciosa: reducir en 1,4 millo-

Cuadro 5. Reducciones regionales estimadas en materia de pobreza y exclusión social (EE2020)

\begin{tabular}{|c|c|c|c|c|c|}
\hline \multirow[b]{2}{*}{$\begin{array}{l}\text { Comunidad } \\
\text { autónoma }\end{array}$} & \multicolumn{4}{|c|}{ (personas) } & \multirow[b]{2}{*}{$\begin{array}{c}\text { Tasa de } \\
\text { cumplimiento }^{4}\end{array}$} \\
\hline & $\begin{array}{l}\text { Población de } \\
\text { referencia } 2009\end{array}$ & $\begin{array}{l}\text { Evolución } \\
2009-2013^{1}\end{array}$ & $\begin{array}{c}\text { Reducción del objetivo } \\
\text { para } 2020^{2}\end{array}$ & $\begin{array}{l}\text { Población del } \\
\text { objetivo } 2020^{3}\end{array}$ & \\
\hline Andalucía & 2.725 .663 & 469.082 & -336.629 & 2.389 .034 & $-1,39$ \\
\hline Aragón & 167.451 & 92.446 & -20.681 & 146.770 & $-4,47$ \\
\hline Asturias & 201.746 & 28.047 & -24.916 & 176.829 & $-1,13$ \\
\hline Baleares & 258.413 & 46.828 & -31.915 & 226.498 & $-1,47$ \\
\hline Canarias & 767.117 & -21.042 & -94.742 & 672.375 & 0,22 \\
\hline Cantabria & 103.816 & 43.844 & -12.822 & 90.994 & $-3,42$ \\
\hline Castilla-La Mancha & 584.493 & 182.609 & -72.187 & 512.306 & $-2,53$ \\
\hline Castilla y León & 556.109 & -44.066 & -68.681 & 487.427 & 0,64 \\
\hline Cataluña & 1.397 .261 & 90.927 & -172.566 & 1.224 .694 & $-0,53$ \\
\hline Ceuta y Melilla & 52.979 & 12.665 & -6.543 & 46.436 & $-1,94$ \\
\hline C. Valenciana & 1.304 .378 & 257.058 & -161.095 & 1.143 .283 & $-1,60$ \\
\hline Extremadura & 382.697 & 6.566 & -47.264 & 335.432 & $-0,14$ \\
\hline Galicia & 679.428 & -15.773 & -83.912 & 595.516 & 0,19 \\
\hline La Rioja & 61.235 & 8.566 & -7.563 & 53.672 & $-1,13$ \\
\hline Madrid & 1.232 .457 & 44.807 & -152.213 & 1.080 .244 & $-0,29$ \\
\hline Navarra & 64.472 & 27.149 & -7.963 & 56.510 & $-3,41$ \\
\hline País Vasco & 315.587 & 48.883 & -38.976 & 276.611 & $-1,25$ \\
\hline R. de Murcia & 480.420 & 16.072 & -59.334 & 421.087 & $-0,27$ \\
\hline Totales España & 11.335 .722 & 1.294 .667 & -1.400 .000 & 9.935 .722 & $-0,92$ \\
\hline
\end{tabular}

1. Variación de la población arope entre 2009 y 2013.

2. Reducción regional proporcional al peso de la región en la población arope total. Su resultado es el objetivo nacional de $-1,4$ millones de personas.

3. Resultado de aplicar la reducción regional (columna 3) a la población de referencia en 2009 (columna 1).

4. Cociente entre las columnas 2 y 3.

Fuente: elaboración propia a partir de INE (2015), Encuesta de Condiciones de Vida. 
nes de personas a la población arope entre 2009 y 2020. El año tomado como referencia ha de ser necesariamente 2009, que es hasta donde retrocede la nueva serie de la ECV (base 2013), sin que, por el momento, se pueda comparar en nuestro país 2013 con 2008, el año inicial de referencia contemplado en la EE2020. Lo que se ha hecho ha sido repartir proporcionalmente la disminución de 1,4 millones de personas en riesgo de pobreza o exclusión social entre las distintas comunidades autónomas, en función de su importancia relativa en el total nacional de dicha población en 2009. Aunque se podría haber utilizado cualquier otro, el criterio empleado para regionalizar el objetivo nacional es coherente con la idea de respetar la integridad del mismo, evitando que se vea mermado, al tiempo que resulta fácil de aplicar e interpretar. En Lois et al. (2013: 235), se reparte de forma homogénea entre las regiones europeas el objetivo global para el total de la UE expresado en términos porcentuales ${ }^{5}$. Por su parte, en el índice regional Europa 2020, un indicador sintético elaborado para comparar el progreso global de la EE2020 en el conjunto de la UE referido a diferentes escalas territoriales (nacional, regional y urbana o rural), se sigue un procedimiento más laborioso debido a la diversidad de regiones e indicadores que lo integran (Athanasoglou y Dijkstra, 2014; Dijkstra y Athanasoglou, 2015) ${ }^{6}$.

De ese modo, la cuarta columna del cuadro 5, denotada mediante un número 3, recoge la reducción requerida en cada autonomía para lograr el objetivo nacional, siendo dicha reducción regional proporcional a su peso poblacional e idéntica en términos porcentuales para cada región y el conjunto nacional, lo cual representa un 12,4\%. Al restar a la población arope de 2009 la reducción así calculada, se obtiene la población Objetivo2020 de cada autonomía. Finalmente, comparando la reducción necesaria con la evolución seguida durante el periodo 2009-2013, se obtiene una medida del grado de cumplimiento de la estrategia hasta el momento, lo cual proporciona una medida relativa del esfuerzo necesario para el cumplimiento del objetivo propuesto.

Como se ha dicho, la reducción necesaria para alcanzar el objetivo en materia de pobreza y exclusión en 2020 representa una disminución del 12,4\% para el total nacional (aplicable a todas las comunidades autónomas) con respecto al valor alcanzado por la población arope en 2009. Esto ya proporciona una primera idea del importante esfuerzo necesario para alcanzar el objetivo, que equivale a casi revertir la tendencia del periodo 2009-2013, donde el incremento del promedio español fue del $11,4 \%$, superado ampliamente por la mayor parte de las regiones. Con todo, la cifra de personas en riesgo de pobreza o exclusión social se situaría cerca de los 10 millones.

5. Estos autores calculan en términos porcentuales lo que representa para el total de la UE la reducción de 20 millones de personas en riesgo de pobreza o exclusión social (un 17\%), asumiendo que todos los territorios deben bajar en ese mismo porcentaje su población arope. Se obtiene así la población objetivo para 2020 correspondiente a cada región.

6. En concreto, el desempeño regional en reducir el riesgo de pobreza o exclusión social se mide a través de la relación entre su distancia a una tasa arope objetivo para 2020 estimada sobre el máximo de esa distancia en todas las regiones. 
Solo las regiones donde la tasa de cumplimiento registra valores positivos (cuadro 5) se encuentran en la senda pactada de reducción de la población en riesgo de pobreza o exclusión. La que más camino ha recorrido es Castilla y León, pues la disminución de su población arope entre 2009 y 2013 representa el $64 \%$ de la reducción requerida. Por el contrario, la región peor posicionada es Aragón, donde el incremento experimentado por la población arope en estos años de crisis multiplica por más de cuatro el objetivo para 2020. Puesto que la mayoría de comunidades ha incrementado su población arope muy por encima de la cifra estimada en la que debería haberse reducido para lograrlo, el esfuerzo requerido en materia de lucha contra la exclusión social se ve multiplicado por dos o incluso por tres en buena parte de ellas.

\section{Conclusiones}

Se podría decir que, por el momento, los resultados obtenidos en España en términos de crecimiento integrador son más bien fruto de la coyuntura económica que de las actuaciones emprendidas por las instituciones nacionales y europeas. La crisis ha empeorado notablemente la situación en materia de empleo y riesgo de exclusión social, alejando sus cifras cada vez más de la meta establecida, mientras que, por el contrario, la escasez de trabajo ha provocado la permanencia y el retorno a los estudios de buena parte de la población joven, lo cual ha mejorado los indicadores educativos. En consecuencia, la tibia actuación de las autoridades nacionales y europeas para corregir la trayectoria opuesta a lo acordado en materia de pobreza y exclusión social no ha logrado ningún fruto. Esta situación es reflejo del escaso grado de compromiso de los gobiernos nacional y europeo con dicho objetivo hasta el momento, encaminados a priorizar la estabilidad macroeconómica y presupuestaria.

La crisis económica ha incrementado el riesgo de pobreza y exclusión social en nuestro país, sin que se haya visto modificada sustancialmente su distribución regional. Solo las comunidades más envejecidas, Galicia y Castilla y León, mejoran su tasa arope entre 2009-2013, debido al descenso de la proporción de pobres entre la población mayor de 65 años. El resto de regiones empeora el riesgo de pobreza o exclusión social con diferente intensidad.

Como se ha visto, la definición del propio objetivo para el conjunto de la UE (sin carácter vinculante) y su traducción a objetivos nacionales (mermados con respecto al global), junto a la ausencia de una dimensión territorial (regional o local), han limitado considerablemente su alcance, presentando también diversas debilidades en relación con el indicador seleccionado: la tasa arope.

El incremento del riesgo de pobreza o exclusión social en España entre 2009 y 2013 representa en un 92\% la reducción consignada en el objetivo nacional en materia de pobreza menos ambicioso ( $-1,4$ millones de personas). Solo tres regiones españolas se encuentran en la dirección adecuada para el logro de dicho objetivo. En cambio, la mayoría de ellas está bastante alejada del objetivo regional estimado, lo que implica un esfuerzo de reducción de la población en riesgo que, como poco, equivale al incremento experimentado de 2009 a 2013. 
Por consiguiente, las posibilidades de lograr la meta acordada son escasas. Aun cuando así fuera, nuestros cálculos estiman que la cifra de población en riesgo de pobreza o exclusión social en España en 2020 no sería muy diferente de las registradas en los primeros años de crisis, puesto que rondaría los 10 millones de personas, resultado que está en sintonía con el pesimismo que la propia UE expresa en su evaluación intermedia de la EE2020: «la UE se ha alejado del objetivo marcado — 96,4 millones de personas en 2020 - sin que haya visos de que esta situación pueda mejorar rápidamente, ya que el número de personas al borde de la pobreza podría seguir rondando los 100 millones de personas en 2020» (Comisión Europea, 2014a: 16).

En el caso español, el diagnóstico y las recomendaciones efectuadas para nuestro país por parte de las instituciones europeas (Comisión Europea, 2015a y 2016; Comité de las Regiones, 2013) señalan la necesidad de actuar con mayor eficacia en la lucha contra la pobreza y la exclusión social, mejorando la gestión y la coordinación entre los distintos niveles de las administraciones públicas, para corregir las divergencias socioeconómicas entre regiones y dotar de una mayor cobertura a los sistemas de protección social. Entre otras medidas, se proponen la homogeneización de los sistemas regionales de rentas mínimas, la ampliación de las prestaciones familiares y de vivienda, junto con una mayor coordinación entre los servicios sociales y de empleo a niveles autonómico y central.

\section{Referencias bibliográficas}

Arriola, Joaquín (2012). «Europa 2020: Una estrategia contra la pobreza en vía muerta». En: FunDACIÓN FOESSA. Exclusión y desarrollo social. Madrid: Fundación FOESSA.

- (2014). La estrategia de la Unión Europea de lucha contra la pobreza. Madrid: Fundación FOESSA. Documento de Trabajo 8.2.

Athanasoglou, Stergios y Dijkstra, Lewis (2014). The Europe 2020 Regional Index [en línea]. Luxemburgo: Publications Office of the European Union. $<$ http://dx.doi.org/10.2788/87940>.

Ayala, Luis (2010). «La pobreza en España: Tendencias y factores de cambio». Revista Española del Tercer Sector, 15, 17-42.

- (2012). «Es la política social la "cenicienta" de la crisis?: Retos y políticas para la inclusión social». En: EAPN. Nuevas propuestas para nuevos tiempos. Madrid: EAPN-España.

CÁRITAS (2013). Europe 2020 Shadow Report: Missing the train for inclusive growth. Time is running out. Bruselas: Caritas Europa.

- (2014). Europe 2020 Shadow Report. Europe 2020: Where are we now and what way forward? 5 years after committing to poverty reduction \& employment growth. Bruselas: Caritas Europa.

COMisión Europea (2003). Informe conjunto sobre la inclusión social en el que se resumen los resultados del examen de los planes nacionales de acción a favor de la inclusión social (2003-2005). COM(2003), 773 final.

- (2010a). Europa 2020: Una estrategia para un crecimiento inteligente, sostenible e integrador. COM(2010), 2020.

- (2010b). La Plataforma Europea contra la Pobreza y la Exclusión Social: Un marco europeo para la cohesión social y territorial. $\operatorname{COM}(2010), 758$ final. 
- (2013a). Comprender las políticas de la Unión Europea. Europa 2020: La estrategia europea de crecimiento. Luxemburgo: Oficina de Publicaciones de la Unión Europea.

- (2013b). Resumen de los avances en la aplicación de las recomendaciones especificas por paises por Estado miembro: Documento de trabajo de los servicios de la Comisión que acompaña al Estudio Prospectivo Anual sobre el Crecimiento para 2014. SWD(2013), 800 final.

- (2014a). Balance de la Estrategia Europa 2020 para un crecimiento inteligente, sostenible e integrador. $\operatorname{COM}(2014), 130$ final.

- (2014b). Preparar la revisión de la Estrategia Europa 2020 para un crecimiento inteligente, sostenible e integrador. Anexos. COM(2014), 130 final.

- (2015a). Recomendación del Consejo relativa al Programa Nacional de Reformas de 2015 de España y por la que se emite un dictamen del Consejo sobre el Programa de Estabilidad de 2015 de España. COM(2015), 259 final.

- (2015b). Resultados de la consulta pública sobre la Estrategia Europa 2020 para un crecimiento inteligente, sostenible e integrador. COM(2015), 100 final.

- (2016). Informe sobre España 2016. SWD(2016), 78 final.

Comité de las Regiones (2013). Fighting poverty and social exclusion: What role for regions and cities? Bruselas: Committee of the Regions.

- (2014). Blueprint for a revised Europe 2020 strategy. Bruselas: Committee of the Regions.

- (2015). $\sigma^{\text {th }}$ CoR Monitoring Report on Europe 2020 and the European Semester. Bruselas: Committee of the Regions.

Cruz Roja (2013). Reflexión creativa: Consecuencias humanitarias de la crisis económica en Europa. Ginebra: Federación Internacional de Sociedades de la Cruz Roja y de la Media Luna Roja.

Dijkstra, Lewis y Athanasoglou, Stergios (2015). The Europe 2020 index: The progress of EU countries, regions and cities to the 2020 targets. Regional Focus, 1/2015.

EAPN (2013). Lifeboat or Life Sentence?: The Troika and emergency assistance programmes and their impact on poverty and social exclusion. Bruselas: European Antipoverty Network (EAPN).

- (2014). Aportaciones de EAPN a la Revisión Intermedia de la Estrategia Europa 2020: ¿Podemos transformar la Estrategia en una herramienta adecuada para cumplir sus promesas de reducción de la pobreza? Bruselas: European Anti-poverty Network (EAPN).

- (2015). El estado de la pobreza. Madrid: EAPN-España.

Eurostat (2015a). Statistics on income, social inclusion and living conditions. EU-SILC Database. <http://ec.europa.eu/eurostat/web/income-and-living-conditions/data/ database $>$.

- (2015b). Smarter, greener, more inclusive?: Indicators to support the Europe 2020 strategy. Luxemburgo: Publications Office of the European Union.

Frazer, Hugh; Guio, Anne; Marlier, Eric; Vanhercke, Bart y Ward, Terry (2014). Putting the fight against poverty and social exclusion at the heart of the EU agenda: A contribution to the Mid-Term Review of the Europe 2020 Strategy. OSE Research Paper, 15.

Fresno, José Manuel; Renes, Víctor y Tsolakis, Andreas (2012). «Estrategia Europa 2020 e inclusión social: Distanciamiento creciente entre objetivos, políticas e instrumentos». Zerbitzuan [en línea], 51, 27-47. <http://dx.doi.org/10.5569/1134-7147/51.02>.

FUNDACIÓN FOESSA (2014). VII Informe sobre exclusión social y desarrollo social en España. Madrid: Cáritas / Fundación FOESSA. 
Hernández Pedreño, Manuel (dir.) (2008). Exclusión social en la Región de Murcia. Murcia: Editum.

- (dir.) (2014). Evolución de la exclusión social en la Región de Murcia: Repercusiones sociales de la crisis. Murcia: Editum.

INE (2015). Encuesta de Condiciones de Vida. INEbase. <http://www.ine.es/jaxiT3/ Tabla.htm? $\mathrm{t}=10011 \& \mathrm{~L}=0>$.

Jurado, Antonio y Pérez, Jesús (2010). «Dimensión territorial de la pobreza en España». Revista Española del Tercer Sector, 15, 43-66.

- (2014). Disparidades entre las comunidades autónomas españolas en el periodo 20072012. Madrid: Fundación FOESSA. Documento de Trabajo 2.9.

Lafuente Lechuga, Matilde y Faura Martínez, Úrsula (2012). «Estudio de la vulnerabilidad a la exclusión social por Comunidades Autónomas (2005-2009)». Investigaciones Regionales, 23, 105-124.

Laparra, Miguel (2010). «El impacto de la crisis en la cohesión social o el surf de los hogares españoles en el modelo de integración de la sociedad líquida». Documentación Social, 158, 97-130.

Laparra, Miguel y Pérez, Begoña (coords.) (2012). Crisis y fractura social en Europa: Causas y efectos en España. Barcelona: Obra Social "la Caixa”. Estudios Sociales, 35.

Leschke, Janine; Theodoropoulou, Sotiria y WatT, Andrew (2012). «How do economic governance reforms and austerity measures affect inclusive growth as formulated in the Europe 2020 Strategy?». En: LeHndorf, Steffen (ed.). A triumph of failed ideas: European models of capitalism in the crisis. Bruselas: European Trade Union Institute (ETUI).

LoIs, Rubén; Feal, Alejandra y PAüL, Valeria (2013). «La dimensión territorial de la Estrategia Europa 2020: Las regiones europeas en la senda oficial para salir de la crisis». Ería, 93, 211-242.

Martínez, Rosa y Navarro, Carolina (2014). Pobreza y privación: Tendencias y determinantes. Madrid: Fundación FOESSA. Documento de trabajo 2.2.

Martínez López, Antonio (2011). «El discurso de la Unión Europea en materia de políticas de empleo y exclusión social: Análisis sociológico de la Estrategia Europea de Empleo». Papers: Revista de Sociologia, 96 (1), 35-54. <http://dx.doi.org/10.5565/rev/papers/v96n1.109>.

MINHAP (2015). Programa Nacional de Reformas de España. Madrid: Ministerio de Hacienda y Administraciones Públicas.

Nolan, Brian y Whelan, Christopher (2011). The EU 2020 Poverty Target. Gini Discussion Paper, 19.

OECD (2014). How's Life in Your Region?: Measuring Regional and Local Well-being for Policy Making [en línea]. París: OECD Publishing. <http://dx.doi.org/10.1787/9789264217416-en>.

Pérez Mayo, Jesús (2013). "Crisis económica y territorio: El impacto de la crisis sobre la desigualdad de rentas en las distintas regiones españolas». Papeles de Economía Española, 135, 36-49.

Unión Europea (2013). «Reglamento (UE) n. o 1304/2013 del Parlamento Europeo y del Consejo de 17 de diciembre de 2013 relativo al Fondo Social Europeo y por el que se deroga el Reglamento (CE) n.o 1081/2006 del Consejo». Diario Oficial de la Unión Europea, L 347/477 (20 diciembre 2013).

Vega, Pilar y Méndez, José (2014). Comparación de los ingresos del trabajo entre la Encuesta de Condiciones de Vida y las fuentes administrativas. Documento de Trabajo 2/2014 del INE.

Ward, Terry y Ozdemir, Erhan (2013). Measuring low work intensity: An analysis of the indicator. ImPRovE Working Paper, 13/09. 